hep-th/0511265

December 2005

\title{
Suggestion of Explicit Field Transformations underlying Misaligned Supersymmetry
}

\author{
Paul H. Frampton \\ Perimeter Institute, 31 Caroline Street, Waterloo, ON N2L 2Y5, Canada \\ and \\ University of North Carolina, Chapel Hill, NC 27599-3255, USA *
}

\begin{abstract}
In order that nonsupersymmetric quiver gauge theories can satisfy naturalness requirements to all orders of perturbation theory, one expects a global symmetry similar to, but different from, supersymmetry. Consistent with the generalized no-go theorem published by Haag et al in 1975, we suggest a generalization of supersymmetry to a misaligned supersymmetry where fermionic generators do not commute with gauge transformations. An explicit form for the corresponding field transformations is suggested.
\end{abstract}

\footnotetext{
${ }^{*}$ Permanent address
} 


\section{Introduction}

It is an old notion that $\mathcal{N}=4$ supersymmetric gauge theory is germane to the generalization of the standard model of particle phenomenology. The $\mathcal{N}=4$ theory has remarkable properties which include ultra violet finiteness and conformal invariance.

Nevertheless, one striking feature of the standard model is the presence of chiral fermions which excludes both $\mathcal{N}=4$ and $\mathcal{N}=2$ extended supersymmetries. Also, although the situation might change, the absence of any experimental support even for $\mathcal{N}=1$ supersymmetry is striking.

This has led to reconsideration of the nonsupersymmetric $\mathcal{N}=0$ case, including $\mathcal{N}=0$ quiver gauge theories with an $U(N)^{n}$ gauge group and matter fields in bifundamental representations. Here one obstacle is a theoretical one in the form of well-known no-go theorems.

Before the discovery of supersymmetry, Coleman and Mandula [1] proved a no-go theorem published in 1967 which stated that the only possible symmetries of the S matrix were products of spacetime and internal symmetries. Shortly after the discovery of supersymmetric theories, Haag, Lopuszanski and Sohnius generalized this to show in a 1975 publication [2] that under certain assumptions, supersymmetry is the only possibility.

No-go theorems can be useful because they provide a set of assumptions some or all of which must be violated in order to make progress. For example, Coleman and Mandula considered only Lie algebras with commutators and not graded algebras with anticommutators. Here, I shall suggest that the way around the generalized no-go theorem of Haag, Lopuszanki and Sohnius is to relax their assumption that the generators of the symmetry commute with gauge transformations. In particular, for a $U(N)^{n}$ quiver gauge theory, we suggest fermionic generators which transform as bi-bifundamentals under $U(N)^{n}$.

In Section II, the symmetry transformations of $\mathcal{N}=4$ Yang-Mills are briefly reviewed. In Section III, the explicit form of a misaligned supersymmetry transformation is suggested, with fermionic generators which transform under the gauge group. Finally in Section IV there is discussion. 


\section{II. $\mathcal{N}=4$ supersymmetry}

Here we collect briefly some well-known facts, for convenience.

The action for $\mathcal{N}=4$ Yang-Mills can be written

$$
\begin{aligned}
S= & \int d^{4} x\left[-\frac{1}{4} F_{\mu \nu a} F^{\mu \nu a}+\frac{1}{2} D_{\mu} \Phi_{i j}^{a} D^{\mu} \Phi_{i j}^{a}+i \bar{\chi}^{a} \gamma \cdot D L \chi^{a}\right. \\
& -\frac{1}{2} i g f_{a b c}\left(\overline{\tilde{\chi}}^{a i} L \chi^{j b} \Phi_{i j}^{c}-\bar{\chi}_{i}^{a} R \tilde{\chi}_{j}^{b} \Phi^{i j c}\right) \\
& \left.-\frac{1}{4} g^{2}\left(f_{a b c} \Phi_{i j}^{b} \Phi_{k l}^{c}\right)\left(f_{a d e} \Phi^{i j d} \Phi^{k l e}\right)\right]
\end{aligned}
$$

where $\mu, \nu=0,1,2,3 ; i, j, k, l=1,2,3 ; L=\frac{1}{2}\left(1+\gamma_{5}\right), R=\frac{1}{2}\left(1-\gamma_{5}\right)$; and $\tilde{\chi}_{i}=C \bar{\chi}^{i T}$ with $C$ the charge conjugation operator.

The action (11) is invariant under the $\mathcal{N}=4$ supersymmetry [3]

$$
\begin{aligned}
\delta A_{\mu}^{a} & =i\left(\bar{\alpha}_{i} \gamma_{\mu} L \chi^{i a}-\bar{\chi}_{i}^{a} \gamma_{\mu} L \alpha^{i}\right) . \\
\delta \Phi_{i j}^{a} & =i\left(\bar{\alpha}_{j} R \tilde{\chi}_{i}^{a}-\bar{\alpha}_{i} R \tilde{\chi}_{i}^{a}+\epsilon_{i j k l} \bar{\alpha}^{k} L \chi^{l a}\right) . \\
\delta L \chi^{i a} & =\sigma_{\mu \nu} F^{\mu \nu a} L \alpha^{i}-\gamma \cdot D \Phi^{i j a} R \tilde{\alpha}_{j}+\frac{1}{2} g f_{a b c} \phi_{b}^{i k} \Phi_{k j}^{c} L \alpha^{j} \\
\delta R \tilde{\chi}_{i}^{a} & =\sigma_{\mu \nu} F^{\mu \nu a} R \tilde{\alpha}_{i}+\gamma \cdot D \Phi_{i j}^{a} L \alpha^{i}+\frac{1}{2} g f_{a b c} \Phi_{i k}^{b} \Phi_{c}^{k j} L \tilde{\alpha}_{j} .
\end{aligned}
$$

where $\alpha^{i}$ transforms as a $\mathbf{4}$ and $\bar{\alpha}_{i}$ as a $\overline{\mathbf{4}}$ under an internal $S U(4)$ symmetry.

The group indices $a, b, c$ run over the dimension of the gauge group $a, b, c=1, \ldots ., d_{G}$. For $G=S U(N)$ or $U(N), d_{G}=\left(N^{2}-1\right)$ or $N^{2}$ respectively. Note that the infintesimal supersymmetry parameter $\alpha^{i}$ is singlet under the gauge group $G$. This assumption will be relaxed for misaligned supersymmetry in the next section. 


\section{Misaligned supersymmetric gauge field theory (MSGFT)}

The name is taken from [4] where string models without supersymmetry were studied, particularly the supertrace conditions necessary for cancellation of ultra violet divergences. The nonsupersymmetric quiver gauge theories introduced in [5] and analyzed further in [6] satisfy such supertrace conditions if all scalars are in bifundamentals [7] so the name "misaligned" supersymmetric gauge field theory (MSGFT) is appropriate. In [4], however, no explicit field transformation underlying misaligned supersymmetry was given and my aim here is to suggest how this may be accomplished.

More recently, in [8], it was discussed how chiral trangle anomalies can be compensated in MSGFT.

A specific MSGFT model is defined by several integers, namely $N$ (the number of coalescing parallel D3 branes in AdS/CFT, also the $N$ in the gauge group $\left.U(N)^{n}\right), n$ (defining the abelian orbifold group $Z_{n}$, also the $n$ in the gauge group $U(N)^{n}$ ); and three integers $A_{1}, A_{2}, A_{3}$ which specify the embedding $Z_{n} \subset S U(4)$ where $S U(4)$ is the internal symmetry of the $\mathcal{N}=4$ case corresponding to replacing the orbifold by a manifold. Note that the fourth integer $A_{4}$ defining the transformation of the 4 of $S U(4)$ is not independent because $A_{4}=-A_{1}-A_{2}-A_{3}(\bmod n)$. In summary, MSGFT models (of the subclass studied in $[5,6])$ are specified by five integers $\left\{N, n, A_{1}, A_{2}, A_{3}\right\}$.

The action for such a MSGFT in the present notation (adapted from $[3,7]$ ) is

$$
\begin{aligned}
S= & \int d^{4} x\left[-\frac{1}{4} F_{\mu \nu a ; r, r} F_{r, r}^{\mu \nu a}+\frac{1}{2} D_{\mu} \Phi_{i j ; r+a_{i}, r}^{a} D^{\mu} \Phi_{i j ; r, r+a_{i}}^{a}+i \bar{\chi}_{r+A_{m}, r}^{a} \gamma \cdot D L \chi_{r, r+A_{m}}^{a}\right. \\
& -\frac{1}{2} i g f_{a b c}\left(\overline{\tilde{\chi}}_{r, r+A_{m}}^{a i} L \chi_{r+A_{m}, r+A_{m}+A_{n}}^{j b} \Phi_{i j ; r+A_{m}+A_{n}, r}^{c}\right. \\
& \left.-\bar{\chi}_{i ; r, r+A_{m}}^{a} R \tilde{\chi}_{j ; r+A_{m}, r-A_{n}}^{b} \Phi_{r-A_{n}, r}^{i j c}\right) \\
& \left.-\frac{1}{4} g^{2}\left(f_{a b c} \Phi_{i j ; r, r+a_{i}}^{b} \Phi_{k l ; r+a_{i}, r+a_{i}+a_{j}}^{c}\right)\left(f_{a d e} \Phi_{r+a_{i}+a_{j}, r+a_{j}}^{i j d} \Phi_{r+a_{j}, r}^{k l e}\right)\right]
\end{aligned}
$$

in which the $a_{i}$ are defined by $a_{i}=A_{2}+A_{3}, a_{2}=A_{3}+A_{1}, a_{3}=A_{1}+A_{2}$; the subscript $r=1,2, \ldots . n$ is a node label; when the two node superscripts are equal it is an adjoint plus singlet of that $U(N)_{r}$; when the two subscripts are unequal it is a bifundamental and the two gauge labels transform under different $U(N)$ gauge groups. 
Now we address the question of what variation of the fields in the action (3) will leave it invariant. Given the field content, the infinitesimal fermionic parameters must transform under the $U(N)^{n}$ gauge group. As a generalization of equations (2), we suggest

$$
\begin{aligned}
& \delta\left(A_{\mu}^{(p)}\right)_{\alpha_{p}}^{\beta_{p}}=i\left(\left[\bar{\alpha}_{i}\right]_{\alpha_{p}, \delta_{p+A_{m}}}^{\beta_{p}, \gamma_{p}} \gamma_{\mu} L\left(\chi^{i\left(p, p+A_{m}\right)}\right)_{\gamma_{p}}^{\delta_{p+A_{m}}}-\left(\bar{\chi}_{i}^{\left(p-A_{m}, p\right)}\right)_{\gamma_{p-A_{m}}}^{\delta_{p}} \gamma_{\mu} L\left[\alpha^{i}\right]_{\alpha_{p}, \delta_{p}}^{\alpha_{p}, \gamma_{p-A_{m}}}\right) \\
& \delta\left(\Phi_{i j}^{\left(p, p+a_{i}\right)}\right)_{\alpha_{p}}^{\alpha_{p+a_{i}}}=i\left(\left[\bar{\alpha}_{j}\right]_{\alpha_{p}, \beta_{p+A_{m}}}^{\alpha_{p+a_{i}}, \beta_{p}} R\left(\tilde{\chi}_{i}^{\left(p, p+A_{m}\right)}\right)_{\beta_{p}}^{\beta_{p+A_{m}}}-\left[\bar{\alpha}_{i}\right]_{\alpha_{p}, \beta_{p+A_{m}}}^{\alpha_{p+a_{i}}, \beta_{p}} R\left(\tilde{\chi}_{j}^{\left(p, p+A_{m}\right)}\right)_{\beta_{p}}^{\beta_{p+A_{m}}}\right. \\
& \left.+\epsilon_{i j k l}\left[\bar{\alpha}^{k}\right]_{\alpha_{p}, \beta_{p+A_{m}}}^{\alpha_{p+a_{i}, \beta_{p}}} L\left(\chi^{l\left(p, p+A_{m}\right)}\right)_{\beta_{p}}^{\beta_{p+A_{m}}}\right) . \\
& \delta\left(L \chi^{i\left(p, p+A_{m}\right)}\right)_{\alpha_{p}}^{\alpha_{p+A_{m}}}=\sigma_{\mu \nu}\left(F^{\mu \nu(p)}\right)_{\beta_{p}}^{\gamma_{p}} L\left[\alpha^{i}\right]_{\alpha_{p} \gamma_{p}}^{\alpha_{p+A_{m}} \beta_{p}} \\
& -\gamma \cdot D\left(\Phi^{i j\left(p, p+a_{i}\right)}\right)_{\beta_{p}}^{\beta_{p+a_{i}}} R\left[\tilde{\alpha}_{j}\right]_{\alpha_{p} \beta_{p+a_{i}}}^{\alpha_{p+A_{m}} \beta_{p}} \\
& +\frac{1}{2} g \epsilon_{\alpha_{p+a_{i}} \beta_{p+a_{i}} \gamma_{p+a_{i}}} \epsilon^{\beta_{p} \gamma_{p} \delta_{p}}\left(\phi^{i k\left(p, p+a_{i}\right)}\right)_{\gamma_{p}}^{\beta_{p+a_{i}}}\left(\Phi_{k j}^{\left(p, p+a_{i}\right)}\right)_{\delta_{p}}^{\gamma_{p+a_{i}}} L\left[\alpha^{j}\right]_{\alpha_{p} \beta_{p}}^{\alpha_{p+A_{m}} \alpha_{p+a_{i}}} \\
& \delta\left(R \tilde{\chi}_{i}^{\left(p-A_{m}, p\right)}\right)_{\alpha_{p-A_{m}}}^{\alpha_{p}}=\sigma_{\mu \nu}\left(F^{\mu \nu(p)}\right)_{\beta_{p}}^{\gamma_{p}} R\left[\tilde{\alpha}_{i}\right]_{\alpha_{p-A_{m}} \gamma_{p}}^{\alpha_{p} \beta_{p}} \\
& +\gamma \cdot D\left(\Phi_{i j}^{\left(p-a_{i}, p\right)}\right)_{\beta_{p-a_{i}}}^{\beta_{p}} L\left[\alpha^{j}\right]_{\alpha_{p-A_{m}} \beta_{p}}^{\alpha_{p} \beta_{p-a_{i}}} \\
& +\frac{1}{2} g \epsilon_{\alpha_{p+a_{i}} \beta_{p+a_{i}} \gamma_{p+a_{i}}} \epsilon^{\beta_{p} \gamma_{p} \delta_{p}}\left(\phi_{i k}^{\left(p, p+a_{i}\right)}\right)_{\gamma_{p}}^{\beta_{p+a_{i}}}\left(\Phi^{k j\left(p, p+a_{i}\right)}\right)_{\delta_{p}}^{\gamma_{p+a_{i}}} R\left[{\tilde{\alpha_{j}}}_{j}\right]_{\alpha_{p-A_{m}} \beta_{p}}^{\alpha_{p} \alpha_{p+a_{i}}}
\end{aligned}
$$

The equations (41) are written so that they reduce to the $\mathcal{N}=4$ equations (2) when the internal $U(N)^{n}$ dependence of the fermionic generators is removed and are written such that the transformation properties under the gauge group $U(N)^{n}$ are consistent for each term in the field transformations (4).

In the limit $A_{m}=a_{i}=0$ and $n=1$, the bifundamentals become adjoints and the couplings in the transformations Eq.(4) reduce to those in Eq.(2); this requirement excludes further (symmetric) cubic couplings in Eq. (41).

We see that the infinitesimal generators $\mathrm{n}$ Eq. (4) must generically be outer products of two bifundamentals under $U(N)^{n}$ although in all terms of (4) this reduces to an outer product of one adjoint with one bifundamental. In the transformation of the $\chi^{i}$ fields I have for definiteness specialized to the case $N=3$ in generalizing the structure constants $f_{a b c}$ of (2) for adjoint representations to the antisymmetric tensors $\epsilon_{\alpha \beta \gamma}$ in (41) for bifundamental representations; for general $N$ one can form $\# 2$ a unique antisymmetric cubic invariant from bifundamentals writable in two equivalent forms

$$
f_{a b c}\left(\lambda^{a}\right)_{i^{\prime}}^{i}\left(\lambda^{b}\right)_{j^{\prime}}^{j}\left(\lambda^{c}\right)_{k^{\prime}}^{k} \Phi_{i}^{i^{\prime}} \Phi_{j}^{j^{\prime}} \Phi_{k}^{k^{\prime}} \quad \text { or } \quad \epsilon^{i j k l m n \ldots x y z} \epsilon_{i^{\prime} j^{\prime} k^{\prime} l m n \ldots x y z} \Phi_{i}^{i^{\prime}} \Phi_{j}^{j^{\prime}} \Phi_{k}^{k^{\prime}}
$$

\footnotetext{
${ }^{\# 2}$ I thank Professor T.W. Kephart for this remark.
} 


\section{Discussion.}

A first issue concerns the no-go theorems of [1,2]. There is no problem with [1] which did not consider fermionic generators and the generalized no-go theorem in [2] implicitly assumes that the fermionic generators are singlets under the gauge group; since this assumption is violated in misaligned supersymmetry, the no-go theorem [2] is inapplicable.

There remain a number of questions to be explored: Does variation under the field transformations (41) really provide an exact symmetry of the action (3)? Do the generators form a closed algebra and the transformations a group? What are the representations of this group? The quiver diagram must form a representation but it may be reducible. It would be interesting to know the irreducible representations. Do MSGFT share properties of supersymmetric gauge theories such as non renormalization theorems? Can a MSGFT be conformally invariant?

It opens the door for research to study $\mathrm{TeV}$ scale conformality models, alternative to $\mathrm{TeV}$ scale supersymmetry. Experiment will enable us to ascertain the approach favored by Nature.

\section{Acknowledgements}

The hospitality of the Perimeter Institute is acknowledged. This research was supported in part by the U.S. Department of Energy under Grant No. DE-FG02-97ER-41036. 


\section{References}

[1] S. Coleman and J.E. Mandula, Phys. Rev. 159, 1251 (1967).

[2] R. Haag, J.T. Lopuszanski and M. Sohnius, Nucl. Phys. B88, 257 (1975).

[3] L. Brink, J. Scherk and J.H. Schwarz, Nucl. Phys. B121, 77 (1977).

[4] K.R. Dienes, Nucl. Phys. B429, 533 (1994). hep-th/9402006.

[5] P.H. Frampton, Phys. Rev. D60, 041901 (1999). hep-th/9812117.

[6] P.H. Frampton and W.F. Shively, Phys. Lett. B454, 49 (1999). hep-th/9902168.

P.H. Frampton and C. Vafa. hep-th/9903226.

P.H. Frampton, Phys. Rev. D60, 085004 (1999). hep-th/9905042; ibid D60, 121901 (1999). hep-th/9907051.

P.H. Frampton, R.N. Mohapatra and S. Suh, Phys. Lett. B520, 331 (2001). hep-ph/0104211.

P.H. Frampton, Mod. Phys. Lett. A18, 1377 (2003). hep-ph/0208044

P.H. Frampton, R.M. Rohm and T. Takahashi, Phys. Lett. B567, 265 (2003). hep-ph/0302074.

[7] X. Calmet, P.H. Frampton and R.M. Rohm, Phys. Rev. D72, 055003 (2005). hep-th/0412176.

[8] E. Di Napoli and P.H. Frampton, to appear. 